\title{
An Overview of the Special Issue on Plant Phenotyping for Disease Detection
}

\author{
Mónica Pineda
}

Citation: Pineda, M. An Overview of the Special Issue on Plant Phenotyping for Disease Detection.

Remote Sens. 2021, 13, 4182

https://doi.org/10.3390/rs13204182

Received: 23 September 2021

Accepted: 16 October 2021

Published: 19 October 2021

Publisher's Note: MDPI stays neutra with regard to jurisdictional claims in published maps and institutional affiliations.

Copyright: (C) 2021 by the author. Licensee MDPI, Basel, Switzerland. This article is an open access article distributed under the terms and conditions of the Creative Commons Attribution (CC BY) license (https:// creativecommons.org/licenses/by/ $4.0 /)$.
Department of Biochemistry and Molecular and Cell Biology of Plants, Estación Experimental del Zaidín, Consejo Superior de Investigaciones Científicas, 18008 Granada, Spain; monica.pineda@eez.csic.es; Tel.: +34-958-181-600

According to the latest United Nations estimates in September 2021, the world's population is now 7.9 billion people and growing. Feeding such a large number of people (and farm animals) presents a challenge for societies and their governments in a world immersed in a process of climate change. Therefore, optimizing agriculture, the main source of vegetables at the base of the food chain, is a key point to ensure the sustainability of the future world. This involves taking a series of measures that increase productivity, quality and sustainability of crops, while minimizing potential environmental risks. Precision agriculture, a management strategy that processes and analyses temporal and spatial data to support efficient management decisions, is born out of this need. Within this framework, non-invasive plant phenotyping imaging techniques are gradually being implemented in agriculture to map constraints in crop fields at proximal and remote scales. In addition, powerful big data algorithms are also required to handle the large complexity and size of the data generated by imaging techniques. A series of articles collected in the Remote Sensing special issue, entitled "Plant phenotyping for disease detection", sheds light on great efforts made by scientists and farmers to carry imaging techniques into effect. The specific goal is to detect infected plants in crop fields, as plant pathogens are one of the most severe constraints limiting crop quality and productivity worldwide. The collection of eight articles provides new insights into this topic, while highlighting the importance of plant phenotyping in modern agriculture, as will be reviewed below.

There are an increasing number of imaging sensors available for diagnosis and plant disease detection, each of them assessing the optical properties of plants within different regions of the electromagnetic spectrum. The most affordable ones, RGB cameras, has been demonstrated to be useful for the automatic evaluation of wheat resistance to Fusarium head blight, one of the most devastating fungal diseases [1]. The novelty of this study resides in the development of an integrated approach for disease severity assessment based on RGB images and mask region convolutional neural networks. The method allowed the high-throughput wheat spike recognition and precision Fusarium head blight infection segmentation under complex field conditions. Affected individual spikes and diseased areas were accurately classified (77.76\% and $98.81 \%$, respectively), being able to distinguish several degrees of disease severity. The approach provides a fast, simple and inexpensive method that will greatly facilitate the breeding of resistant cultivars.

Hyperspectral sensors have been widely shown to be useful for detecting stressed plants, and their price has become more affordable over the years, earning a place in their own right in the world of remote sensing. Six out of eight articles in this special issue use them to successfully diagnose infected plants. However, hyperspectral sensors provide a large, complex amount of spectral information that requires a dimensional reduction to identify the most informative wavelengths to diagnose a particular disease. To this end, feature selection methods were applied to hyperspectral data to successfully identify the most important wavelengths, in order to discriminate between healthy and diseased peanut plants infected with Athelia rolfsii, the causal agent of peanut stem rot [2]. Selected wavelengths can be used to design optical sensors specific for stem rot detection. 
Nonetheless, this methodology can be adapted to identify spectral signatures of disease in other plant-pathogen systems.

Hyperspectral cameras can obtain the reflectance spectrum for each pixel in the image of a scene, thus adding another dimension to the recorded data. Moreover, they can be implemented in a great variety of phenotyping platforms, such as robots or unmanned aerial vehicles. Consequently, their use in combination with big data tools for pathogen diagnosis has increased in latest years. Indeed, hyperspectral reflectance data could detect potato tubers infested with the nematode Meloidogyne luci with a 100\% accuracy rate, being on a level with the real-time PCR [3]. Even tubers with no visible infestation symptoms could be successfully diagnosed. Furthermore, peeling potatoes is not required for the detection of latent infestation, making the method fast and efficient to avoid the uncontrolled spread of the pest.

Disease resistance evaluation of seedlings in nurseries and greenhouses is a laborious and time-consuming process, whereby efficiency and accuracy could be greatly improved by using high-throughput phenotyping methods. As an example, the classification models (support vector machines) applied to hyperspectral images of loblolly pines seedlings were able to discriminate between healthy and diseased fusiform rust plants with an accuracy up to $77 \%$ [4]. Authors found that this technique is a viable and efficient method for the detection of disease incidence and could be applied in resistance-screening centres. Phytoplasmas, such those causing grapevine yellows, are serious diseases affecting vine growing areas around the world as no curative in-field treatments are available. Thus, finding detection methods based on plant phenotyping seems to be a good option to avoid the spread of the infection. Disease detection models based on hyperspectral reflectance images and radial-basis function networks have been designed for greenhouse vine plants infected with phytoplasmas, thereby achieving satisfying classification accuracies up to $96 \%$ when sorting symptomatic and healthy plants [5]. Moreover, the most significant wavelengths to detect the infection could be identified, which can be used to design more affordable multispectral systems.

As shown previously, finding the most informative wavelengths for a given hostpathogen system is crucial when dealing the complex information provided by the hyperspectral sensors [2,5], as well as finding suitable vegetation indices (VIs) to maximise the differences between healthy and infected plants among the large collection of available VIs in the literature. Once again, big data algorithms come to the aid of researchers. The M statistic method were used to find the most appropriate VIs to accurately distinguish different diseases that affect tomato crops, such as bacterial spot, target spot and tomato yellow leaf curl [6].

It is still possible to get even more out of a hyperspectral image, as for example, the texture features (TF). Texture in an important attribute of an image providing information on the spatial arrangement of the intensity of the pixels, enabling the differentiation of different regions based on their TFs. Infected plants show alterations in their external characteristics, such as leaf colour or morphology that change TFs. Thus, plants suffering wheat yellow rust, one of the most destructive diseases in wheat production, could be classified by support vector machines with higher accuracy when combining spectral features (optimal wavelengths and VIs) with TFs [7]. The approach was used to successfully map the yellow rust lesions on wheat leaves with different damage levels.

Thermography is one of the most widely used techniques in remote sensing due to its versatility and ease of use. Thermal cameras record the emitted radiation in the infrared region of the spectrum, providing images representing temperature values per pixel. Leaf temperature is highly correlated to stomatal conductance, which is a helpful indicator of the physiological status of plants. Thus, thermography can feasibly replace conventional measurements of stomatal conductance, providing data in short periods of time. However, it is crucial to take into account other factors that affect leaf surface temperature, such as air temperature, humidity, wind speed and canopy structure angle, especially when measurements are done in the field. Thermal indices incorporate these corrections into the 
images, allowing the use of this technique in the field. In addition, thermal indices can be correlated with other important crop features, such as soil moisture, hydric potential, net photosynthesis and even yield. Therefore, thermography, by itself or in combination with other image sensors, is widely used for plant phenotyping and disease diagnosis, as recently reviewed [8].

There are other sensors that could be used for plant disease diagnose, such as multispectral fluorescence imaging cameras. However, these sensors require specific light sources to excite fluorescence, which limit their application in the field. This problem has been partially solved with the application of lasers, or alternatively, with passive methodologies based on sun-induced fluorescence. Although the number of high-throughput plant phenotyping platforms is growing, their high cost and the shortage of qualified personnel are limiting factors that can prevent their successful implementation. A long way has been travelled, but much more research is needed to make plant phenotyping a reality in the present and future agriculture.

Funding: This research received no external funding.

Institutional Review Board Statement: Not applicable.

Informed Consent Statement: Not applicable.

Data Availability Statement: Not applicable.

Conflicts of Interest: The authors declare no conflict of interest.

\section{References}

1. Su, W.-H.; Zhang, J.; Yang, C.; Page, R.; Szinyei, T.; Hirsch, C.D.; Steffenson, B.J. Automatic evaluation of wheat resistance to fusarium head blight using dual mask-RCNN deep learning frameworks in computer vision. Remote Sens. 2021, 13, 26. [CrossRef]

2. Wei, X.; Johnson, M.A.; Langston, D.B.; Mehl, H.L.; Li, S. Identifying optimal wavelengths as disease signatures using hyperspectral sensor and machine learning. Remote Sens. 2021, 13, 2833. [CrossRef]

3. Žibrat, U.; Gerič Stare, B.; Knapič, M.; Susič, N.; Lapajne, J.; Širca, S. Detection of root-knot nematode Meloidogyne luci infestation of potato tubers using hyperspectral remote sensing and real-time PCR molecular methods. Remote Sens. 2021, 13, 1996. [CrossRef]

4. Pandey, P.; Payn, K.G.; Lu, Y.; Heine, A.J.; Walker, T.D.; Acosta, J.J.; Young, S. Hyperspectral imaging combined with machine learning for the detection of fusiform rust disease incidence in loblolly pine seedlings. Remote Sens. 2021, 13, 3595. [CrossRef]

5. Bendel, N.; Backhaus, A.; Kicherer, A.; Köckerling, J.; Maixner, M.; Jarausch, B.; Biancu, S.; Klück, H.-C.; Seiffert, U.; Voegele, R.T.; et al. Detection of two different grapevine yellows in Vitis vinifera using hyperspectral imaging. Remote Sens. 2020, $12,4151$. [CrossRef]

6. Abdulridha, J.; Ampatzidis, Y.; Qureshi, J.; Roberts, P. Laboratory and UAV-based identification and classification of tomato yellow leaf curl, bacterial spot, and target spot diseases in tomato utilizing hyperspectral imaging and machine learning. Remote Sens. 2020, 12, 2732. [CrossRef]

7. Guo, A.; Huang, W.; Ye, H.; Dong, Y.; Ma, H.; Ren, Y.; Ruan, C. Identification of wheat yellow rust using spectral and texture features of hyperspectral images. Remote Sens. 2020, 12, 1419. [CrossRef]

8. Pineda, M.; Barón, M.; Pérez-Bueno, M.-L. Thermal imaging for plant stress detection and phenotyping. Remote Sens. 2021, 13, 68. [CrossRef] 